\title{
Comparing endoscopic ultrasound-guided core biopsies of solid pancreatic and extrapancreatic lesions: a large single-operator experience with a new fine-needle biopsy needle
}

\author{
Abdul Haseeb, Linda Jo Taylor, Douglas G. Adler \\ University of Utah School of Medicine, Salt Lake City, Utah, USA
}

\section{Abstract}

\section{Introduction}

In the era of molecular profiling and personalized oncology, there is an increasing emphasis on obtaining histological samples with tissue cores for molecular and ancillary testing [1,2]. Since

Department of Internal Medicine, Division of Gastroenterology and Hepatology, University of Utah School of Medicine, Salt Lake City, Utah, USA

\section{Conflict of Interest: None}

Correspondence to: Douglas G. Adler MD, FACG, AGAF, FASGE, Professor of Medicine, Director of Therapeutic Endoscopy, Director, GI Fellowship Program, Gastroenterology and Hepatology, University of Utah School of Medicine, Huntsman Cancer Center, 30N 1900E 4R118, Salt Lake City, Utah 84132, USA, e-mail: Douglas.adler@hsc.utah.edu

Received 4 April 2018; accepted 22 August 2018; published online 24 September 2018

DOI: https://doi.org/10.20524/aog.2018.0313 its introduction in 1992, endoscopic ultrasound (EUS) fineneedle aspiration (FNA) has shown increasingly better diagnostic accuracy with improved needle designs over the years. However, the aspirate obtained by FNA fails to provide information on tissue architecture and limits the ability to perform grade differentiation, molecular profiling, and other ancillary testing.

Fine-needle biopsy (FNB) needle technology has evolved rapidly during the last decade, paralleling the therapeutic indications for EUS. FNB specimens ideally provide preserved tissue architecture and a larger amount of tissue for any ancillary testing. A new Franseen-geometry, three-plane

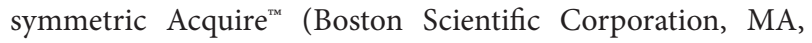
USA) FNB needle showed promising results in recent studies with small sample sizes. Most of the previous EUS-FNB studies looked primarily at solid pancreatic lesions, whereas very few extrapancreatic lesions were evaluated. Historically, the diagnostic accuracy of EUS-FNA is lower for extrapancreatic lesions than for pancreatic lesions, while only limited data are available from the use of EUS-FNB [3]. We performed a 
retrospective study with a larger number of patients, comparing the performance of this new Franseen needle design in both pancreatic and extrapancreatic solid lesions.

\section{Patients and methods}

We evaluated a longitudinal retrospective cohort of patients with solid masses or lesions needing EUS-guided core biopsy at the University of Utah between May 2016 and November 2017. The Institutional Review Board at the University of Utah approved the study.

The medical records of all identified patients were reviewed manually. Patients with cystic lesions based on EUS and other cross-sectional imaging data were excluded from the study. Data points included, but were not limited to age, sex, location of the lesion, size of the lesion, endoscopic ultrasound findings, type of tissue acquisition (transesophageal, transgastric, transduodenal and transrectal), tissue acquisition techniques (slow pull, saline in the needle, fanning vs. suction), needle gauge, number of passes, technical success and final histopathologic diagnosis. Needle visibility/echogenicity was graded on a scale of 1-4, with 1 being highest and 4 being lowest. Technical success was defined as successful acquisition of the tissue with an FNB needle for adequate interpretation by a pathologist.

EUS-FNB with the Acquire ${ }^{\mathrm{Tm}}$ Endoscopic Ultrasound Fine Needle Biopsy Device (Boston Scientific Corporation, Natick MA, USA) was performed using linear echoendoscopes (Fig. 1,2). All EUS-FNB procedures were performed by a single endosonographer (DGA) with over 15 years of experience at performing high-volume EUS. The technique used for tissue acquisition was to insert the needle into the target lesions and slowly remove the stylet manually during needle actuations ("slow-pull" technique). The same technique was used exclusively in this study for the sake of uniformity. Rapid onsite evaluation (ROSE) by a board-certified cytopathologist was performed in all cases. Pathologic material and reporting records were also reviewed for each case to confirm the number of needle passes needed to achieve diagnostic adequacy, the presence or absence of diagnostic material, and whether a definitive diagnosis could be derived from the specimen obtained.

\section{Statistical analysis}

Statistical analysis was performed using SPSS version 25 (SPSS Inc, Chicago, Ill). Baseline characteristics of the cohort, descriptive statistics, and continuous variables were presented as mean \pm standard deviation.

\section{Results}

A total of 132 patients, 87 (66\%) male and 45 (34\%) female were included in this study. The mean age of the study

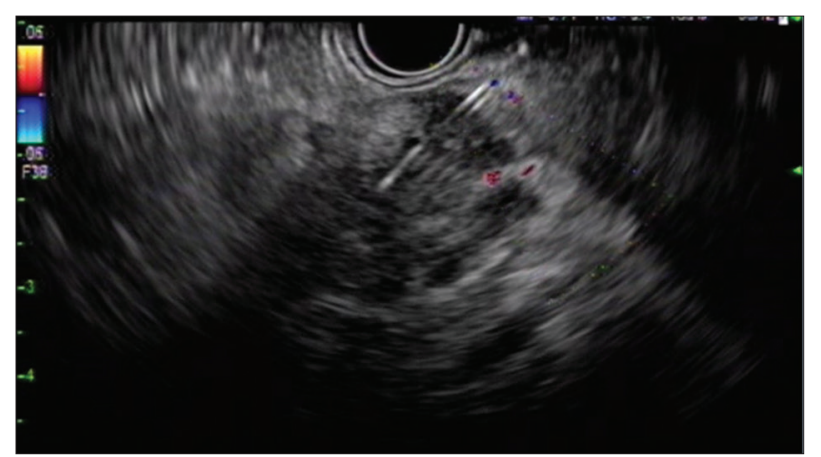

Figure 1 Endoscopic ultrasound image of a 19-G fine-needle biopsy needle used to obtain a core biopsy from a solid pancreatic head mass

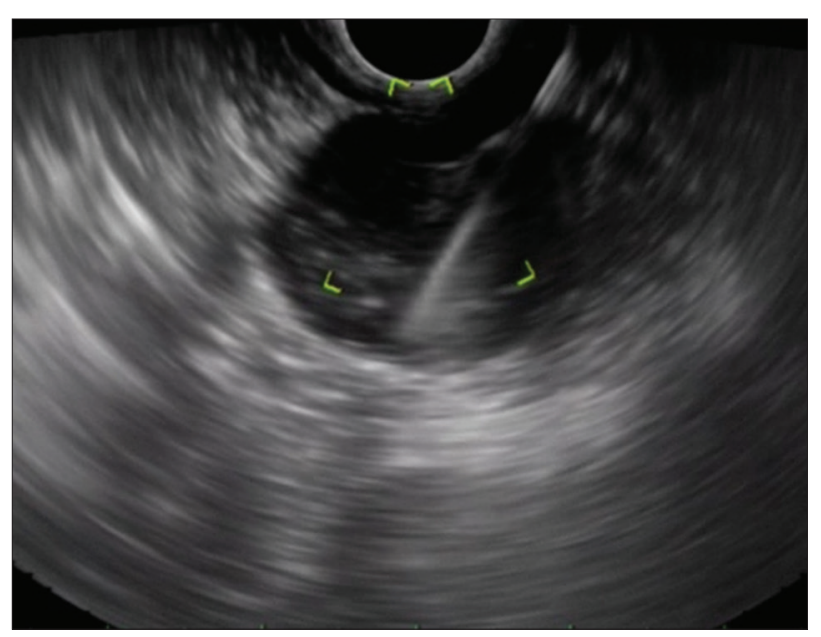

Figure 2 Endoscopic ultrasound image of a 22-G fine-needle biopsy needle used to obtain a core biopsy from an extrapancreatic, mediastinal lymph node

population was $63.5 \pm 13$ years (Table 1 ). This was the first EUS biopsy of the target lesion in all patients.

Ninety-nine (73.3\%) of the biopsied lesions were located in the pancreas and $36(26.7 \%)$ were extrapancreatic in location. Of the pancreatic lesions, $60(60.6 \%)$ were located in the head of the pancreas, $20(20.2 \%)$ in the body, $12(12.1 \%)$ in the tail, $4(4 \%)$ in the uncinate process, and $3(3 \%)$ in the neck of the pancreas. Three different gauges of Acquire ${ }^{\mathrm{Tx}}$ biopsy needle were used: $130(96.3 \%)$ lesions were biopsied with a $22 \mathrm{G}$ needle, $3(2.2 \%)$ with a $19 \mathrm{G}$ needle, and $2(1.5 \%)$ with a $25 \mathrm{G}$ needle. The echogenicity of the needle was graded as 1 in all cases.

The transgastric approach was most commonly used, with $67(49.6 \%)$ lesions accessed via this approach, followed by the transduodenal in 64 lesions $(47.4 \%)$, transesophageal in $3(2.2 \%)$, and transrectal in $1(0.7 \%)$ (Table 2$)$.

ROSE was performed by a board-certified pathologist in all cases. Our on-site pathologists used small samples of the core biopsy to make cytology slides to assess cellularity and adequacy. The majority of patients needed more than one pass to obtain an adequate specimen: 19 (14.4\%) patients needed 1 pass, $68(51.5 \%)$ two passes, 35 (26.5\%) three passes, $9(6.8 \%) 4$ passes, and 1 patient needed 5 passes. 
Table 1 Demographics and location of solid lesions studied by endoscopic ultrasound

\begin{tabular}{lc}
\hline Total number of patients & 132 \\
Total number of core biopsies obtained & 135 \\
Sex & \\
Male & $87(66 \%)$ \\
Female & $45(34 \%)$ \\
Lesion location & \\
Pancreatic & $99(73.3 \%)$ \\
Extrapancreatic & \\
Stomach & $4(2.9 \%)$ \\
Rectum & $2(1.4 \%)$ \\
Mediastinal lymph node & $4(2.9 \%)$ \\
Celiac lymph node & $8(5.9 \%)$ \\
Porta hepatis lymph node & $6(4.4 \%)$ \\
Peripancreatic lymph node & $8(5.9 \%)$ \\
Retroperitoneal lymph node & $2(1.4 \%)$ \\
Adrenal gland & $1(0.7 \%)$ \\
Location of pancreatic lesions & \\
Head & $60(60.6 \%)$ \\
Body & $20(20.2 \%)$ \\
Tail & $12(12.1 \%)$ \\
Uncinate & $4(4 \%)$ \\
Neck & $3(3 \%)$ \\
\hline
\end{tabular}

Table 2 Endoscopic ultrasound (EUS) fine-needle biopsy needle gauge, acquisition modality, number of passes needed and final diagnosis

\begin{tabular}{lc}
\hline Needle gauge & \\
$22 \mathrm{G}$ & $130(96.3 \%)$ \\
$25 \mathrm{G}$ & $2(1.5 \%)$ \\
$19 \mathrm{G}$ & $3(2.2 \%)$ \\
\hline EUS modality & \\
Transesophageal & $3(2.2 \%)$ \\
Transgastric & $67(49.6 \%)$ \\
Transduodenal & $64(47.4 \%)$ \\
Transrectal & $1(0.7 \%)$ \\
Number of passes needed per & \\
patient & $19(14.4 \%)$ \\
1 & $68(51.5 \%)$ \\
2 & $35(26.5 \%)$ \\
3 & $9(6.8 \%)$ \\
4 & $1(0.8 \%)$ \\
5 & \\
Need for re-biopsy & $3(2.3 \%)$ \\
Final diagnosis & \\
Pancreatic adenocarcinoma & $74(56 \%)$ \\
Gastrointestinal stromal tumors & $6(4.5 \%)$ \\
Neuroendocrine tumors & $12(9.1 \%)($ all pancreatic) \\
Lymphoma & $4(3.0 \%)$ \\
Benign lesions & $15(11.5 \%)(5 / 15$ \\
Pancreatic metastases & pancreatic) \\
Non-pancreatic metastatic lesions & $7(5.3 \%)$ \\
Non-pancreatic adenocarcinoma & $7(5.3 \%)$ \\
Non-diagnostic specimen & $6(4.5 \%)$ \\
Solid pseudo papillary tumor of & $1(0.8 \%)($ non-pancreatic) \\
pancreas & $1(0.8 \%)$ \\
\hline
\end{tabular}

The mean number of passes needed for an adequate sample was $2.28 \pm 0.7$. Similar numbers of passes were needed for pancreatic $(2.3 \pm 0.8)$ compared to extrapancreatic $(2.3 \pm 0.6)$ lesions. Only 3 of 132 patients, with two pancreatic lesions and one extrapancreatic, needed re-biopsy at a later date. The overall diagnostic accuracy of the biopsied tissue was $97.7 \%$. Biopsy samples from 128/132 patients (97\%) were split for histological and cytological evaluation. Of the remaining 4 patients, two biopsies (1.5\%) were sent for histological evaluation only and two for cytological evaluation only. No immediate adverse events were reported.

The final pathological diagnoses of the obtained biopsies were notable for pancreatic adenocarcinoma in 74 (56\%), benign etiologies in $15(11.5 \%)$, neuroendocrine tumors in $12(9.1 \%)$, pancreatic metastases in $7(5.3 \%)$, gastrointestinal stromal tumors in $6(4.5 \%)$, lymphoma in $4(3 \%)$, nonpancreatic metastases in 7 (5.3\%), and non-pancreatic adenocarcinoma in $6(4.5 \%)$. One of the specimens obtained was deemed non-diagnostic and the patient declined a re-biopsy. Benign etiologies included changes consistent with pancreatitis in 5 patients and benign adenopathy in 10 patients.

Comparing pancreatic to extrapancreatic sites, despite the $100 \%$ diagnostic yield based on ROSE, two pancreatic lesions and one extrapancreatic needed re-biopsy at a later date as the final pathology was indeterminate. Overall, FNB of the pancreatic lesions had a diagnostic accuracy of $97.9 \%$, comparable to the $97.2 \%$ for extrapancreatic lesions (Table 3).

\section{Discussion}

Our retrospective study suggests that the use of core biopsy needles is an effective modality for tissue acquisition from both pancreatic and extrapancreatic solid lesions, and is the first study to specifically assess the value of a Franseen-tip needle when sampling extrapancreatic lesions FNA needles have an variable accuracy of $61 \%$ to $92 \%$ in pancreatic lesions, with significant heterogeneity among published studies [4-6]. Over the last decade, multiple Tru-Cut biopsy needles (e.g., Quick-Core: Cook medical, Limerick, Ireland) have been developed, improving sample procurement and offering a higher histological tissue yield $[7,8]$. With a diagnostic yield estimated to be between $52 \%$ and $95 \%$, these needles were not superior to conventional FNA needles [9-13]. EUS-FNA quality indicators were proposed in 2015 , with a diagnostic rate of malignancy for all pancreatic masses $\geq 70 \%$ and a sensitivity for malignancy among patients with pancreatic cancer $\geq 85 \%$ [14].

Recently there has been a paradigm shift in tertiary centers from EUS-FNA/Tru-Cut to EUS-FNB for tissue procurement; this was due to the improved diagnostic accuracy and the availability ofROSE [12]. Recent data on several new FNB needles have shown impressive tissue acquisition and a high rate of solid tissue cores available for more detailed analysis by pathology [15-17]. Earlier studies have shown that these needles are at least as good as standard FNA needles, while 
Table 3 Comparison of endoscopic ultrasound fine-needle biopsies of pancreatic and extrapancreatic lesions

\begin{tabular}{lcc}
\hline Characteristic & $\begin{array}{c}\text { Pancreatic } \\
\text { solid lesions }\end{array}$ & $\begin{array}{c}\text { Extrapancreatic } \\
\text { solid lesions }\end{array}$ \\
\hline Number of lesions biopsied & $99(73.3 \%)$ & $36(26.7 \%)$ \\
$\begin{array}{l}\text { Mean number of passes } \\
\begin{array}{l}\text { Diagnostic yield based on } \\
\text { ROSE }\end{array}\end{array}$ & $2.3 \pm 0.8$ & $2.3 \pm 0.6$ \\
$\begin{array}{l}\text { Patients needing re-biopsy } \\
\text { after the final pathology } \\
\text { read }\end{array}$ & 2 & $100 \%$ \\
$\begin{array}{l}\text { Diagnostic accuracy } \\
\text { Immediate complications }\end{array}$ & $97.9 \%$ & 1 \\
\hline ROSE, rapid on-site evaluation & None & None \\
\hline
\end{tabular}

they offer additional tissue for special stains with fewer needle passes overall [18]. While FNB sampling may obviate the need for ROSE, some in the cytology community still favor FNA over FNB [19]. Practices with regards to the need for ROSE in patients undergoing FNB remain unstandardized.

FNB sampling provides intact cores that provide better characterization of malignancy and improve the diagnostic accuracy for benign lesions. With extrapancreatic lesions (especially gastrointestinal stromal tumors, lymphoma, and neuroendocrine tumors), histology is crucial for reaching a diagnosis, as cytology alone cannot uniformly provide adequate architecture and cellular configuration [20,21]. In a prior study at our center, we compared the older reversebevel FNB needle to a standard FNA needle and found no significant difference in core tissue acquisition and diagnostic accuracy [22]. These findings were restated in a recent metaanalysis by Bang et al [11] which showed no significant difference between the reverse-bevel ProCore needles (22G/25G, Cook Endoscopy) and standard FNA needles (22 G/25 G) in terms of tissue acquisition and diagnostic accuracy. A few studies noted improved diagnostic yield with FNB of extrapancreatic lesions when compared to pancreatic lesions [23,24].

With the rapid evolution in needle technology, a new needle using a Franseen geometrical tip design (Acquire, Boston Scientific, Natick, MA) was recently introduced to maximize tissue capture and minimize fragmentation. In a recent study of 59 patients, using this needlefor biopsy of only pancreatic solid lesions showed a high procurement yield and 98.3\% diagnostic accuracy [25] This is comparable to our diagnostic accuracy of $97.9 \%$ for pancreatic and 97.2\% for extrapancreatic lesions. Most of the FNB needle studies published to date focused primarily on pancreatic lesions, while only a few extrapancreatic lesions were evaluated. Our study is the first to specifically evaluate extrapancreatic solid lesions using the novel Franseen Acquire $^{\text {tM }}$ needle. Of the lesions evaluated in our study, $26.7 \%$ were extrapancreatic in location and FNB of these lesions showed comparable diagnostic accuracy without any adverse events.
The mean number of passes needed in previous studies ranged from 2.8-3.5 [16,18]. This is higher than the mean number of $2.28 \pm 0.7$ passes needed in our study. Similar numbers of passes were needed for pancreatic $(2.3 \pm 0.8)$ and for extrapancreatic $(2.3 \pm 0.6)$ lesions. A study by Bang et al [26] reported a $3.3 \%$ rate of complications using a similar Franseen-design needle. Studies using 22/25 G SharkCore (Covidien, Sunnyvale, USA) needles showed increased bleeding associated with transduodenal biopsies, not observed in our study [27]. However, we encountered no procedure-related complications, similarly to Mitri et al [25].

Some limitations of our study should be noted, including its retrospective nature, single-center and single-operator design, with a possibility of selection bias. This study involved tissue acquisition by a highly experienced endosonographer, limiting its applicability to a real world environment. Given the lack of literature in this area, validated scoring systems were not used for cytology and histology specimens.

In this large, single-center study, EUS-FNB performed with a novel Franseen-type biopsy needle proved to be an effective modality for tissue acquisition from all solid lesions. We showed a very high histological and diagnostic yield, with equal effectiveness in both pancreatic and extrapancreatic lesions. Further evaluation with large, prospective, multicenter studies is needed to evaluate optimal needle diameter, the number of passes needed based on the biopsy site, and the need for ongoing ROSE.

\section{Summary Box}

\section{What is already known:}

- Fine-needle aspiration (FNA) has variable diagnostic accuracy and fails to provide information on tissue architecture

- The diagnostic accuracy with FNA is lower for extrapancreatic lesions than for pancreatic lesions

- Live donor LT (LDLT) has become the most promising alternative for the expansion of the organ pool for adult patients with HCC, but indications and criteria are still subject to debate

\section{What the new findings are:}

- This is the first study to specifically assess the value of a Franseen tip needle FNB for the sampling of extrapancreatic lesions

- All three needle sizes (19 G, 22 G and 25 G) had similar histological yield

- The novel Franseen-type needle showed equal effectiveness in both pancreatic and extrapancreatic lesions, with $>97 \%$ diagnostic accuracy 


\section{References}

1. Au TH, Wang K, Stenehjem D, Garrido-Laguna I. Personalized and precision medicine: integrating genomics into treatment decisions in gastrointestinal malignancies. J Gastrointestinal Oncol 2017;8:387-404.

2. Chantrill LA, Nagrial AM, Watson C, et al. Precision medicine for advanced pancreas cancer: the individualized molecular pancreatic cancer therapy (IMPaCT) trial. Clin Cancer Res 2015;21:2029-2037.

3. Dumonceau JM, Deprez PH, Jenssen C, et al. Indications, results, and clinical impact of endoscopic ultrasound (EUS)-guided sampling in gastroenterology: European Society of Gastrointestinal Endoscopy (ESGE) Clinical Guideline - Updated January 2017. Endoscopy 2017;49:695-714.

4. Chen G, Liu S, Zhao Y, Dai M, Zhang T. Diagnostic accuracy of endoscopic ultrasound-guided fine-needle aspiration for pancreatic cancer: a meta-analysis. Pancreatology 2013;13:298-304.

5. Yoshinaga S, Suzuki H, Oda I, Saito Y. Role of endoscopic ultrasound-guided fine needle aspiration (EUS-FNA) for diagnosis of solid pancreatic masses. Dig Endosc 2011;23(Suppl 1):29-33.

6. Nayar MK, Paranandi B, Dawwas MF, et al. Comparison of the diagnostic performance of 2 core biopsy needles for EUS-guided tissue acquisition from solid pancreatic lesions. Gastrointest Endosc 2017;85:1017-1024.

7. Varadarajulu S, Bang JY, Hebert-Magee S. Assessment of the technical performance of the flexible 19-gauge EUS-FNA needle. Gastrointest Endosc 2012;76:336-343.

8. Larghi A, Verna EC, Ricci R, et al. EUS-guided fine-needle tissue acquisition by using a 19-gauge needle in a selected patient population: a prospective study. Gastroint Endosc 2011;74:504-510.

9. DeWitt J, Cho CM, Lin J, et al. Comparison of EUS-guided tissue acquisition using two different 19-gauge core biopsy needles: a multicenter, prospective, randomized, and blinded study. Endosc Int Open 2015;3:E471-E478.

10. Thomas T, Kaye PV, Ragunath K, Aithal G. Efficacy, safety, and predictive factors for a positive yield of EUS-guided Trucut biopsy: a large tertiary referral center experience. Am J Gastroenterol 2009;104:584-591.

11. Bang JY, Hawes R, Varadarajulu S. A meta-analysis comparing ProCore and standard fine-needle aspiration needles for endoscopic ultrasound-guided tissue acquisition. Endoscopy 2016;48:339-349.

12. Attili F, Petrone G, Abdulkader I, et al. Accuracy and interobserver agreement of the Procore ${ }^{\mathrm{Tw}} 25$ gauge needle for endoscopic ultrasound-guided tissue core biopsy. Dig Liver Dis 2015;47:943-949.

13. Kandel P, Tranesh G, Nassar A, et al. EUS-guided fine needle biopsy sampling using a novel fork-tip needle: a case-control study. Gastrointest Endosc 2016;84:1034-1039.
14. Wani S, Wallace MB, Cohen J, et al. Quality indicators for EUS. Gastrointest Endosc 2015;81:67-80.

15. Adler DG, Muthusamy VR, Ehrlich DS, et al. A multicenter evaluation of a new EUS core biopsy needle: Experience in 200 patients. Endosc Ultrasound 2018.

16. Attili F, Rimbas M, Fantin A, et al. Performance of a new histology needle for EUS-guided fine needle biopsy: A retrospective multicenter study. Dig Liver Dis 2018;50:469-474.

17. Larsen MH, Fristrup CW, Detlefsen S, Mortensen MB. Prospective evaluation of EUS-guided fine needle biopsy in pancreatic mass lesions. Endosc Int Open 2018;6:E242-E248.

18. Tian L, Tang AL, Zhang L, et al. Evaluation of $22 \mathrm{G}$ fine-needle aspiration (FNA) versus fine-needle biopsy (FNB) for endoscopic ultrasound-guided sampling of pancreatic lesions: a prospective comparison study. Surg Endosc 2018;32:3533-3539.

19. Yan L, Ikemura K, Park JW. Utility of core biopsy with concurrent ROSE FNA in the diagnosis of pancreatic tumor-does the biopsy add any diagnostic benefit? Diagn Cytopathol 2018;46:154-159.

20. Wahnschaffe U, Ullrich R, Mayerle J, Lerch MM, Zeitz M, Faiss S. EUS-guided Trucut needle biopsies as first-line diagnostic method for patients with intestinal or extraintestinal mass lesions. Surg Endosc 2009;23:2351-2355.

21. Hashimoto S, Taguchi H, Higashi M, et al. Diagnostic efficacy of liquid-based cytology for solid pancreatic lesion samples obtained with endoscopic ultrasound-guided fine-needle aspiration: Propensity score-matched analysis. Dig Endosc 2017;29:608-616.

22. Witt BL, Adler DG, Hilden K, et al. A comparative needle study: EUS-FNA procedures using the HD ProCore() and EchoTip((R)) 22-gauge needle types. Diagn Cytopathol 2013;41:1069-1074.

23. Vanbiervliet G, Napoleon B, Saint Paul MC, et al. Core needle versus standard needle for endoscopic ultrasound-guided biopsy of solid pancreatic masses: a randomized crossover study. Endoscopy 2014;46:1063-1070.

24. Aadam AA, Wani S, Amick A, et al. A randomized controlled cross-over trial and cost analysis comparing endoscopic ultrasound fine needle aspiration and fine needle biopsy. Endosc Int Open 2016;4:E497-E505.

25. Mitri RD, Rimbas M, Attili F, et al. Performance of a new needle for endoscopic ultrasound-guided fine-needle biopsy in patients with pancreatic solid lesions: A retrospective multicenter study. Endosc Ultrasound 2017.

26. Bang JY, Hebert-Magee S, Hasan MK, Navaneethan U, Hawes R, Varadarajulu S. Endoscopic ultrasonography-guided biopsy using a Franseen needle design: Initial assessment. Dig Endosc 2017;29:338-346.

27. Rodrigues-Pinto E, Jalaj S, Grimm IS, et al. Impact of EUS-guided fine-needle biopsy sampling with a new core needle on the need for onsite cytopathologic assessment: a preliminary study. Gastrointest Endosc 2016;84:1040-1046. 